

\title{
Multiply scattered waves through a spatially random medium : entropy production and depolarization
}

\author{
Dominique Bicout, C. Brosseau
}

\section{To cite this version:}

Dominique Bicout, C. Brosseau. Multiply scattered waves through a spatially random medium: entropy production and depolarization. Journal de Physique I, 1992, 2 (11), pp.2047-2063. 10.1051/jp1:1992266 . jpa-00246685

\section{HAL Id: jpa-00246685 https://hal.science/jpa-00246685}

Submitted on 1 Jan 1992

HAL is a multi-disciplinary open access archive for the deposit and dissemination of scientific research documents, whether they are published or not. The documents may come from teaching and research institutions in France or abroad, or from public or private research centers.
L'archive ouverte pluridisciplinaire HAL, est destinée au dépôt et à la diffusion de documents scientifiques de niveau recherche, publiés ou non, émanant des établissements d'enseignement et de recherche français ou étrangers, des laboratoires publics ou privés. 


\title{
Multiply scattered waves through a spatially random medium : entropy production and depolarization
}

\author{
Dominique Bicout $\left({ }^{1}\right)$ and Christian Brosseau $\left({ }^{2}\right)$ \\ (') Max Planck Institut für Festkörperforschung, Hochfeld Magnet Labor, CNRS, B.P. 166, \\ 38042 Grenoble Cedex 09, France \\ ( ${ }^{2}$ CERMO, Université Joseph Fourier, B.P. 87, 38402 Saint-Martin-d'Hères Cedex, France \\ (Received 12 February 1992, accepted in final form 31 July 1992)
}

\begin{abstract}
Résumé. - Dans cette étude, on considère les phénomènes de dépolarisation et de décohérence d'un faisceau d'ondes planes incident, d'état pur de polarisation et d'état arbitraire de cohérence, par interaction avec un milieu diffusant désordonné. Par des arguments de symétrie et un principe d'entropie maximum, nous déduisons la forme de la matrice de Mueller caractérisant le milieu diffusant qui est en accord avec le calcul explicite basé sur l'équation de Bethe-Salpeter traitée dans l'approximation de la diffusion. Le résultat principal exprime les degrés de polarisation et de cohérence spatiale en fonction du nombre de diffusions. Deux faits saillants sont à noter. Le premier exprime la décroissance exponentielle de la production d'entropie due à l'irréversibilité du processus de dépolarisation, en fonction du nombre de diffusions. Le second indique que la dépolarisation complète d'un faisceau incident polarisé linéairement par des diffuseurs de type Rayleigh nécessite davantage de diffusions (facteur 2) que pour une polarisation circulaire.
\end{abstract}

\begin{abstract}
This paper deals with the depolarization and decoherence effects of an incident pure state of polarization and of arbitrary state of coherence by a linear scattering medium which changes randomly with position. Using symmetry arguments and a maximum entropy principle we deduce the general form of the Mueller matrix describing the scattering medium which is consistent with the explicit computation done in the context of the Bethe-Salpeter equation handled in the diffusion approximation. The main result expresses the output degree of polarization and degree of spatial coherence as a function of the number of scattering events. From these results, two main conclusions can be drawn. The first is that the entropy production per scattering due to the irreversible process of depolarization is an exponentially decreasing function of the number of scattering events. The second result obtained is that full depolarization of linearly polarized light by Rayleigh scatterers requires more scattering events (typically a factor-of-2) than are required for a circularly polarized lightwave.
\end{abstract}

\section{Introduction.}

Studies of scattering of optical waves by inhomogeneous media are currently attracting a wide research interest, in connection with condensed matter physics (e.g. weak Anderson localization [1-2]), propagation of electromagnetic waves under the sea or in the atmosphere [3], and numerous others fields (e.g. image reconstruction [4], information retrieval [5]). In this respect, the subject of polarization correlations if of primary importance. Multiple 
scattering of light from inhomogeneities in optically dense media randomizes the state of polarization, however the polarization memory effect that has been recently evidenced (both theoretically [6-7] and confirmed experimentally [8] soon afterwards) suggests that some information about the state of polarization of the incident beam is not irretrievably lost by multiple scattering. A point of further interest is to characterize the depolarization of optical waves propagating through a random multiple scattering medium. A wave propagating in such a medium becomes rapidly depolarized but one knows little about the characteristic spatial scale on which this process proceeds. This question is important as regards the validation of the scalar diffusion theory [2] to describe the transport properties of multiply scattered light. This raises also the question of the mechanism of depolarization and its relation to the scattering medium. In trying to understand depolarization, it is useful to consider two main approaches. The selective absorption of polarization states is the first approach [9]. The second approach preserves total flux and induces depolarization by decorrelation of the phases and amplitudes of the electric field components [9] ; it is essentially an entropic effect arising from the irreversible evolution of the polarization state during scattering. The present paper is mostly devoted to this latter approach and we will argue that depolarization of light by multiple scattering is connected to a process of entropy production which falls off exponentially with the number of scattering events. The key observation underlying the work reported here is that this result can be derived from a maximum entropy principle.

Our own interest is to investigate the scattering of electromagnetic waves of pure state of polarization and arbitrary state of coherence from a medium which fluctuates in space. Example of such medium would be a collection of randomly oriented scattering particles suspended in a liquid. The purpose of the present work is to calculate the dependence of the polarization and coherence characteristics (Stokes vector, degree of polarization and degree of spatial coherence) upon the number of scattering events. Besides, we will briefly consider an application of this formalism to a technique of photon-correlation spectroscopy known as diffusing wave spectroscopy (DWS) [10] which is relevant to probe the nature of dynamic correlations within dense random dielectric media (e.g. colloidal suspensions).

We emphasize here that we will not inquire into the change in the spectrum of light arising on propagation of light through a spatially random medium which has been treated by Wolf et al. [11], neither on the effects of weak localization in the backscattering of polarized light from a medium containing randomly distributed Rayleigh scatterers [1].

To treat this problem, we use the theory of partial coherence. Such an approach based on the theory of stochastic processes has been initiated largely by Wolf [12-13]. This coherency matrix approach permits us to characterize both the energy transport and the entropy of the electromagnetic field: the intensity is simply the trace of the coherency matrix while the radiation entropy defined following Von Neumann is analytically related to the degree of polarization of the field [9]. Note that the radiation entropy with which we shall be concerned here is of different nature than the spectral entropy derived from Planck's formula [14].

The remainder of the paper is organized as follows. Section 2 is devoted to the formulation of the problem and outlines the elementary concepts in terms of which the problems of depolarization and decoherence will be approached throughout the paper. Section 3 outlines the principle of our entropic approach. Then, section 4 investigates the entropy production associated with multiple scattering of light using the Bethe-Salpeter equation and deals with the dependence of polarization characteristics (i.e. degrees of polarization and of spatial coherence, light-intensity autocorrelation function) with the number of scattering events. Some comments about Freund's prediction [5] that the Stokes vector of an incident pure state of polarization can be fully reconstructed after total depolarization by multiple scattering are discussed in section 5. Finally, several concluding remarks are presented in section 6 . 


\section{Preliminary considerations.}

Let us begin with a brief outline of the main assumptions and background information required for the development of the theory.

2.1 THE INCIDENT FIELD. - Let $E_{j}(\mathbf{r}, t)(j=x, y)$ the two orthogonal components of the electric vector of the incident wave in a plane perpendicular to the direction of propagation (of unit vector $\mathbf{e}_{z}$ ), $\mathbf{r}$ is a position vector of a typical point in space and $t$ is the time. Then, the transverse electric vector writes : $\mathbf{E}=E_{x} \mathbf{e}_{x}+E_{y} \mathbf{e}_{y}$, where $\mathbf{e}_{x}$ and $\mathbf{e}_{y}$ are orthogonal unit vectors in said plane $\left(\mathbf{e}_{j} \cdot \mathbf{e}_{z}=0, \mathbf{e}_{i} \cdot \mathbf{e}_{j}=\delta_{i j}(i, j=x, y)\right)$ with $\left(\mathbf{e}_{x}, \mathbf{e}_{y}, \mathbf{e}_{z}\right)$ forming a right-handed triad of real vectors. Suppose next that this plane-wave field is quasimonochromatic of narrow spectral range centered around $\omega_{0}$. We also assume that the field is statistically homogeneous and stationary, at least in the wide sense. We may characterize the second-order spatial coherence properties of the field by its cross-spectral density tensor $W$ (also termed the coherency matrix) [12] of components : $W_{i j}\left(\mathbf{r}_{1}, \mathbf{r}_{2}\right)=W_{i j}\left(\mathbf{r}_{1}-\mathbf{r}_{2}\right)=\left\langle E_{i}\left(\mathbf{r}_{1}\right) E_{j}^{*}\left(\mathbf{r}_{2}\right)\right\rangle$ at two points whose location is specified by position vectors $\mathbf{r}_{1}$ and $\mathbf{r}_{2}$; the asterisk denoting complex conjugation. The angular brackets denote the average taken over the ensemble that characterizes the statistical properties of the incident field. By definition $\mathbf{W}$ is Hermitian and non-negative definite. For later purpose, we recall some basic polarization properties of the field. The degree of polarization $P$ of the light is given by :

$$
P=\left(1-4 \frac{\operatorname{det}(\mathbf{W})}{(\operatorname{tr}(\mathbf{W}))^{2}}\right)^{1 / 2},
$$

as a function of the two rotational invariants of $\mathbf{W}$. The average Stokes parameters are obtained from $\mathbf{W}$ with : $\left\langle S_{k}\right\rangle=\operatorname{tr}\left(W \sigma_{k}\right)$, where $\sigma_{k}$ is the usual notation for the Pauli matrices ( $\sigma_{0}$ being the $2 \times 2$ unit matrix). The two-level decomposition [9] of any partially polarized light into a completely unpolarized wave and a completely polarized wave which are independent of each other allows us to write :

$$
\mathbf{W}=\frac{1}{2}\left\langle S_{0}\right\rangle \boldsymbol{\sigma}_{0}+\sum_{k=1}^{3}\left\langle S_{k}\right\rangle \boldsymbol{\sigma}_{k}
$$

If we choose the axes of our Cartesian coordinate system along $\mathbf{e}_{x}$ and $\mathbf{e}_{y}$, then

$$
\begin{aligned}
\left\langle S_{0}\right\rangle=\left\langle\left|E_{x}\right|^{2}+\left|E_{y}\right|^{2}\right\rangle, \quad\left\langle S_{1}\right\rangle= & \left\langle\left|E_{x}\right|^{2}-\left|E_{y}\right|^{2}\right\rangle, \\
& \left\langle S_{2}\right\rangle=\left\langle E_{y} E_{x}^{*}+E_{x} E_{y}^{*}\right\rangle, \quad\left\langle S_{3}\right\rangle=i\left\langle E_{y} E_{x}^{*}-E_{x} E_{y}^{*}\right\rangle .
\end{aligned}
$$

These four parameters form the components of the $4 \times 1$ Stokes vector. Stokes parameters are discussed at length in references $[12,15]$; however it is useful to bear in mind their physical interpretation in terms of linear combinations of intensities [16]. It should be emphasized that $\left\langle S_{0}\right\rangle,\left\langle S_{1}\right\rangle,\left\langle S_{2}\right\rangle$, are invariant under the conjugation operation $E_{j} \rightarrow E_{j}^{*} j=x, y$ whereas $\left\langle S_{3}\right\rangle$ changes sign by this transformation. Barakat [16] made also interesting comments on the similarities and differences when one considers the Stokes parameters with respect to time coordinates rather than spatial. Note that an alternative formulation of $P$ in terms of the average Stokes parameters is : $P=\left(\sum_{k=1}^{3}\left(\frac{\left\langle S_{k}\right\rangle}{\left\langle S_{0}\right\rangle}\right)^{2}\right)^{1 / 2}$ It may be viewed as an order parameter indicating the degree to which the wavefield is ordered. A well-known geometric representation of the Stokes parameters is given by the so-called Poincaré sphere $\Sigma_{P}^{2}[9,12]$ of radius 
$P$ (i.e. SO(3) symmetry of the order parameter) in three dimensional space with axes $\frac{\left\langle S_{k}\right\rangle}{\left\langle S_{0}\right\rangle}$. this is the set of states of polarization where the degree of polarization is $P$. This geometrical description based on the convexity property of states [9] bears some similarity to that encountered in the analysis of spin $1 / 2$ [17]. The convex set of states possesses two strata : one of rank 2 corresponding to mixed states (partially polarized) located inside the ball $\Sigma_{P}^{3}$ and the other of rank 1 corresponding to pure states (totally polarized) on the surface $\Sigma_{1}^{2}$. Further, we introduce the complex degree of spatial coherence of the components $E_{x}$ and $E_{y}[12]$ by the expression :

$$
\mu_{x y}(\mathbf{r})=\frac{W_{x y}(\mathbf{r})}{\left(W_{x x}(0)\right)^{1 / 2}\left(W_{y y}(0)\right)^{1 / 2}}=\frac{\left\langle S_{2}\right\rangle+i\left\langle S_{3}\right\rangle}{\left(\left\langle S_{0}\right\rangle^{2}-\left\langle S_{1}\right\rangle^{2}\right)^{1 / 2}},
$$

the normalization in (3) ensures that $0 \leqslant\left|\mu_{x y}\right| \leqslant P \leqslant 1$. The extreme case when $\mu_{x y}=0$ represents spatial incoherence ; the other extreme case $\left|\mu_{x y}\right|=1$ represents spatial coherence between the two field components.

The entropy associated with $W$ is usually taken to be the Von Neumann measure $S(\mathbf{W})=-\operatorname{tr}(\mathbf{W} \ln (\mathbf{W}))$. It takes a simple analytical expression [9] when $\operatorname{tr}(\mathbf{W})=1$ which is the case to be considered here :

$$
S(P)=-\ln (s(P)), \text { with } \quad s(x)=\frac{1}{2}(1+x)^{\frac{1+x}{2}}(1-x)^{\frac{1-x}{2}}
$$

This expression is reminiscent to the above-mentioned two-level decomposition and was derived from equation (2) via the spectral decomposition theorem [9]. For $x$ varying between 0 and $1, s(x)$ takes values between $1 / 2$ and $1, s(x)$ is a bijective strictly increasing function : its inverse exists and is noted $s^{-1}$. Two remarks are of importance. First, the entropy in (4) depends only on $P$ and not on the detailed state of polarization : for instance, it is the same for both linear and circular polarizations. Second, it satisfies the inequalities: $S(P=1) \leqslant S \leqslant S(P=0)$.

2.2 The scattering medium. - We will assume that the scattering medium is linear, isotropic, and non-magnetic occupying a finite volume $\Omega$ (of arbitrary shape and orientation) in free space, and that the spatial fluctuations of its dielectric susceptibility $\mu_{i j}(r)$ tensor are statistically homogeneous and stationary (at least in the wide sense). We restrict our treatment to considering a non-absorbing medium. The effect due to a weak absorption can be easily introduced into our formalism and will be considered in a subsequent work. Typical realization of such medium would be a collection of discrete pointlike scattering centers whose sizes is small compared to the wavelength. We also assume that the time fluctuations of the scatterers are sufficiently slow relative to the period of the field oscillations so that the scattering medium behaves as it is essentially time-invariant (i.e. adiabatic approximation). The usual boundary conditions require continuity of the magnetic field $\mathbf{H}$ and tangential electric field at every discontinuity surface.

From the above assumptions, we may characterize the dielectric susceptibility of the medium by $\mu_{\imath j}(\mathbf{r})=\mu(\mathbf{r}) \delta_{l j}\left(\delta_{i j}\right.$ being the Kronecker symbol), of zero mean and white-noise correlation function :

$$
\left\langle\mu\left(\mathbf{r}_{1}\right) \mu\left(\mathbf{r}_{2}\right)\right\rangle=u \delta\left(\mathbf{r}_{1}-\mathbf{r}_{2}\right) \underset{0}{\text { when }} \quad \mathbf{r}_{1} \in \Omega, \mathbf{r}_{2} \in \Omega
$$

where $u$ is a constant and $\delta(\mathbf{r})$ the Dirac delta function. 
No assumption is made regarding the thermodynamic state of the scattering medium. We will consider only weak disorder (far from the Anderson transition) such that the elastic mean free path $\ell \equiv \frac{6 \pi}{u k_{0}^{4}}$ is much larger than the wavelength of the radiation (i.e. $k_{0} \ell \gg 1$ ), $k_{0}=\omega_{0} / c$ is the vacuum wave number associated with the frequency $\omega_{0}, c$ being the speed of light in vacuo : consequently, the wavefield propagation may be described by a classical diffusion process [2]. Finally, we assume that the fluctuations of the medium and the fluctuations of the incident field are statistically independent.

2.3 SYMMETRY RELATIONSHIPS FOR SCATTERING OF POLARIZED LIGHT, - The input Stokes vector $S_{l}$ and output $S$ tokes vector to the scattering medium $S_{o}$ are related by a linear relation of the form : $\mathbf{S}_{\mathbf{o}}=\mathbf{M S} \mathbf{S}_{l}$, where $\mathbf{M}$ is the $4 \times 4$ Mueller real-valued matrix characterizing the scattering. A number of restrictions are placed at the outset on the form of the M-matrix depending upon the symmetry and reciprocity requirements. Perrin [19] was the first to give a detailed study of the number of independent parameters (among the sixteen matrix elements of M) which are necessary for specifying the polarization characteristics of light scattered by an arbitrary medium. Following the analysis made by Perrin, the forward (or backward) axial scattering by a symmetrical medium (e.g. identical particles having spherical symmetry) involves only three coefficients ; moreover the Mueller matrix is diagonal. We note in passing that further refinements for a variety of symmetry properties of the matrices $\mathbf{M}$ describing the reflection and transmission of polarized radiation by a slab of randomly oriented particles have been treated by Van de Hulst [20] and later by Hovenier [21]. In a statistically isotropic threedimensional medium, there is full $C_{\infty v}$ symmetry around the propagation direction. In twodimensions, the symmetry is reduced to $C_{2 v}$. Consequently the Mueller matrix is not diagonal in two dimensions but block diagonal as proved by Freund [22].

\section{Maximum entropy principle.}

In this section, we evaluate the elements of the Mueller matrix describing the multiple scattering medium by an argument of maximum entropy. We proceed as follows. In view of the symmetry arguments presented in the previous section and the fact that multiple scattering is non dissipative, the general form of the Mueller matrix $\mathbf{M}$ can be written as :

$$
\mathbf{M}=\left[\begin{array}{cccc}
1 & 0 & 0 & 0 \\
0 & m_{11} & 0 & 0 \\
0 & 0 & m_{22} & 0 \\
0 & 0 & 0 & m_{33}
\end{array}\right]
$$

The entropy production per scattering reads as :

$$
\Delta S(n)=S(P(n+1))-S(P(n))=\hat{h}(n),
$$

where $P(n)$ denotes the degree of polarization after $n+1$ scattering events, $S(P)$ being given by equation (4) and the superscript $\wedge$ indicates that $\hat{h}$ depends on the particular state of polarization. Then the total entropy production after $n+2$ scatterings is given by :

$$
\Delta S=S(P(n+1))-S(P(0))=\sum_{j=0}^{n} \hat{h}(j)=\ln \left(\frac{s(P(0))}{s(P(n+1))}\right) .
$$

The function $\hat{h}(x)$ is taken to be a monotonically decreasing function from $\hat{h}(0)$ down to zero ; this is to expected from the theory of irreversible thermodynamics [18]. It can be shown that 
the candidate function $\hat{h}(x)$ must also belong to the metric space $\mathrm{L}^{2}$ and satisfy the condition $\hat{h}^{\prime \prime}(x)>0$ where the prime indicates differentiation with respect to $x$. It is necessary to postulate the functional form of $\hat{h}(x)$ : we have chosen to work out the function $\hat{h}(x)=\psi \exp (-\chi x)$ which meets the above requirements.

Next, we consider an incident pure state of polarization which is linearly polarized $\left(P(n+1)=m_{11}(n)\right)$. From equation (8), we get

$$
s\left(m_{11}\right)=\exp \left(-\sum_{j=0}^{n} \hat{h}(j)\right)=\exp \left(-\psi\left(\frac{1-\exp (-\chi n)}{1-\exp (-\chi)}\right)\right) .
$$

An equivalent expression is

$$
m_{11}(n)=s^{-1}\left(A^{j=0} \sum^{n-1} B^{j}\right)
$$

where we have set for notational convenience $A=\exp (-\psi)$ and $B=\exp (-\chi)$. We call attention to the important fact that $B$ can be written from equation (7) as $B=1-S(P(1)) / \ln (2)$ : consequently $B$ is fully determined by double scattering. Moreover when maximum entropy is achieved (i.e. in the limit $n \rightarrow \infty$ ), we require that $A^{\frac{1}{1-B}}=\frac{1}{2}$; i.e. $\psi=\ln (2)(1-\exp (-\chi))$. The final result is :

$$
m_{11}(n)=s^{-1}\left(2^{\left(B^{n}-1\right)}\right) .
$$

This equation allows the successive orders of iteration to be expressed in terms of the sole parameter $B$. It is worth noting that the same formula will apply for a pure state which is circularly polarized (i.e. $\left.P(n+1)=m_{33}(n)\right)$ with a change in the value of $B$. Note that this method is quite general and may be used for more involved Mueller matrix, but it does not tell us what kind of trial functions $\hat{h}(x)$ are to be used. This simulation can incorporate any function $\hat{h}(x)$ that satisfies the physical constraints.

\section{Light depolarization and decoherence by a scattering medium : the Bethe-Salpeter equation approach}

The problem now shifts to the explicit calculation of the dependence of the degrees of polarization and of spatial coherence in function of the number of scattering events when the incident plane wavefield is of the type described in section 2 and the medium fulfills the assumptions stated above. Indeed, one can evaluate exactly the Mueller matrix elements $m_{i i}(n)$ by the Bethe-Salpeter equation handled in the ladder approximation (see Appendix A). This will serve our purpose to justify the use of the entropic approach developed in the above section. We emphasize that this derivation is valid over distances greater than the elastic mean free path. As demonstrated by Stephen and Cwilich [23], the problem of evaluating the coherency matrix $\mathbf{W}$ reduces to a matrix eigenvalue problem. We leave the details of formulae in the Appendices A and B.

4.1 DEPOLARIZATION BY A TIME-INVARIANT SCATTERING MEDIUM. - We consider an incident (labeled i) pure state of polarization and arbitrary degree of spatial coherence, of unit intensity normally on the half-space $z>0$; its Stokes vector writes as follows:

$$
\mathrm{S}_{l}=\left[\begin{array}{l}
\left\langle S_{0}\right\rangle=\left\langle\left|E_{x}\right|^{2}\right\rangle+\left\langle\left|E_{y}\right|^{2}\right\rangle=1 \\
\left\langle S_{1}\right\rangle=\left\langle\left|E_{x}\right|^{2}\right\rangle-\left\langle\left|E_{y}\right|^{2}\right\rangle \\
\left\langle S_{2}\right\rangle=\left\langle E_{x}^{*} E_{y}+E_{x} E_{y}^{*}\right\rangle \\
\left\langle S_{3}\right\rangle=i\left\langle E_{x}^{*} E_{y}-E_{x} E_{y}^{*}\right\rangle
\end{array}\right] .
$$


In the limit of weak scattering limit, the linear response of the scattering medium is determined by the ensemble averaged covariance satisfying the Bethe-Salpeter equation (Eq. (A1)). The derivation of the correlation function of the field by time-independent Green's functions techniques has been largely discussed [23, 30, 41-42]. Following this method, we obtain the expression for the output (labeled o) Stokes vector :

$$
\mathbf{S}_{\mathrm{o}}=\left[\begin{array}{r}
\left\langle\left|G_{x x}\right|^{2}\right\rangle+\left\langle\left|G_{x y}\right|^{2}\right\rangle \\
\left\langle S_{1}\right\rangle\left(\left\langle\left|G_{x x}\right|^{2}\right\rangle-\left\langle\left|G_{x y}\right|^{2}\right\rangle\right) \\
\left\langle S_{2}\right\rangle\left(\left\langle G_{y y}^{*} G_{x x}\right\rangle+\left\langle G_{y x}^{*} G_{x y}\right\rangle\right) \\
\left\langle S_{3}\right\rangle\left(\left\langle G_{y y}^{*} G_{x x}\right\rangle-\left\langle G_{y x}^{*} G_{x y}\right\rangle\right)
\end{array}\right]
$$

where the $G_{i j}$ 's denote the retarded Green's function (Eq. (A6)) and the suffices $x, y$ label components with respect to the Cartesian coordinate system chosen. Since absorption has been ignored, we normalize $S_{0}$ with respect to $\left\langle\left|G_{x x}\right|^{2}\right\rangle+\left\langle\left|G_{x y}\right|^{2}\right\rangle$. Then, equations (A9) allow us to write $S_{0}$ in the form:

$$
\mathbf{S}_{\mathrm{o}}=\left[\begin{array}{c}
1 \\
\left\langle S_{1}\right\rangle \\
\left\langle S_{2}\right\rangle \\
\left\langle S_{3}\right\rangle(n) \\
\langle(n)
\end{array}\right]
$$

with $f(n)=\frac{3(7 / 10)^{n}}{2+(7 / 10)^{n}}$ and $g(n)=\frac{3(1 / 2)^{n}}{2+(7 / 10)^{n}} ; n+1$ being the number of scattering events. These expressions are very similar to those derived by Freund [6] (his Eq. (11)), albeit in a different fashion. It is readily verified from equations $(12,14)$ that the Mueller matrix of the scattering medium is of the form given by equation (6). The fact that $m_{11}=m_{22}$ is further substantiated by the property of rotational invariance of the degree of polarization.

Having specified $S_{0}$, it follows from equation (14) that the output degree of polarization is given by :

$$
P_{0}=f(n)\left\{\left\langle S_{1}\right\rangle^{2}+\left\langle S_{2}\right\rangle^{2}+\left\langle S_{3}\right\rangle^{2}\left(\frac{5}{7}\right)^{2 n}\right\}^{1 / 2},
$$

which involves three independent parameters. Combining equations (3) and (14), the output degree of spatial coherence writes:

$$
\mu_{x y}^{(o)}=\mu_{x y}^{(i)} f(n)\left(\frac{\left\langle S_{2}\right\rangle+i\left\langle S_{3}\right\rangle\left(\frac{5}{7}\right)^{n}}{\left\langle S_{2}\right\rangle+i\left\langle S_{3}\right\rangle}\right)\left(\frac{1-\left\langle S_{1}\right\rangle^{2}}{1-\left\langle S_{1}\right\rangle^{2}(f(n))^{2}}\right)^{1 / 2}
$$

As they stand, equations (15-16) show that the output degree of polarization (i.e. of spatial coherence) will, in general, differ from the input degree of polarization (i.e. of spatial coherence) because of the effect of the scattering medium. These equations are in accordance with the fact that single scattering (i.e. $n=0$ ) by particles having spherical symmetry preserves the state and degree of polarization [19-21].

For concreteness, it is worthwhile to specialize formulae (15-16) to some special cases of interest. For instance, an input linear polarization state $\left(\mathbf{E}=\mathbf{e}_{x}\right)$ has for output Stokes vector $\left[\begin{array}{c}1 \\ f(n) \\ 0 \\ 0\end{array}\right]$, degree of polarization $P_{0}=f(n)$ which is a monotonically decreasing function of the 
number of scattering (see Fig. 1) and a degree of spatial coherence equal to zero. Similarly, for an input right-handed circular polarization state $\left(\mathbf{E}=\frac{1}{\sqrt{2}}\left(\mathbf{e}_{x}-i \mathbf{e}_{y}\right)\right)$, one gets : $\left[\begin{array}{c}1 \\ 0 \\ 0 \\ g(n)\end{array}\right]$, $P_{\mathrm{o}}=g(n)$ and $\left|\mu_{x y}^{(0)}\right|=\left|\mu_{x y}^{(i)}\right| P_{\mathrm{o}}$. Then, the two functions $f$ and $g$ have clear physical meanings. Numerical calculations are shown in figure 1. Upon comparing $f$ and $g$ in figure 1 , we see that these functions have not the same decay (i.e. $f(n)>g(n)$ ). The parameter $\nu=\frac{\left(\left\langle S_{1}\right\rangle^{2}+\left\langle S_{2}\right\rangle^{1 / 2}\right)}{\left\langle S_{3}\right\rangle}$ which represents the ratio of the linear to circular polarization has been also displayed in figure 1 ; the exponential increase indicates that as the degree of polarization decreases, the polarization ellipse flattens towards the major axis. This fact is also expressed by saying that depolarization of linearly polarized light requires more scattering events than are required for a circularly polarized light. Bourret [24] made a similar comment in his study of propagation of light in a medium with a stochastic refractive index. In this insightful paper, he found by using a random perturbation treatment that the depolarization characteristic length associated with multiple scattering is for linearly polarized light exactly twice the value for circularly polarized light. From the numerical expressions of the functions $f$ and $g$ we find this ratio to be in the range $\left[\frac{\ln (0.5)}{\ln (0.7)}=1.94(n \rightarrow \infty), 2.10(\right.$ low $\left.n)\right]$, i.e. very close to the value found by Bourret.

For $n>1$, the process of depolarization cannot be assimilated to an isotropic contraction of the Poincare sphere $\Sigma_{1}^{2}$ but induces a symmetry breaking (i.e. the symmetry of $\operatorname{SO}(3)$ is



Fig. 1. - Degree of polarization of scattered light as a function of the number of scatterings $n$ (Eq. (15)) for an input pure state of linear parallel polarization (solid line), right circular polarization (dashed line). The inset depicts the dependence of the normalized parameter $\frac{\nu(n)}{\nu(0)}$ on $n$. 
broken). To discuss the physical significance of these results, we have also plotted (Fig. 2) the entropy associated with different states of polarization using equation (4). The variation of the entropy production $\Delta S(n)=S(P(n+1))-S(P(n))$ with the number of scattering events (Fig. 3) is well represented by an exponential decay $\Delta S(n)=\psi \exp (-\chi n)$, with $\psi$ and $\chi$ depending on the particular state of polarization. This form is consistent with results obtained from equation (11). For example, $m_{11}$ (resp. $m_{33}$ ) given by equation (11) was numerically tested assuming that the exact value is given by $f(n)$ (resp. $g(n)$ ). From such calculations, we find that the fitting parameter $B$ was equal to within $4 \%$ of the exact value. This behavior of the entropy production already expresses in a way the irreversible nature of the process considered. For large values of $n$ (say, $n \geqslant 10$ ), the entropy of radiation is unaffected by further scattering, it defines the steady state of maximum entropy $(S(P=0)=\ln (2))$, attainable by multiple scattering. This is of course the standard sort of argument from statistical physics $[14,25]$. Relatively litle is known about this transition from

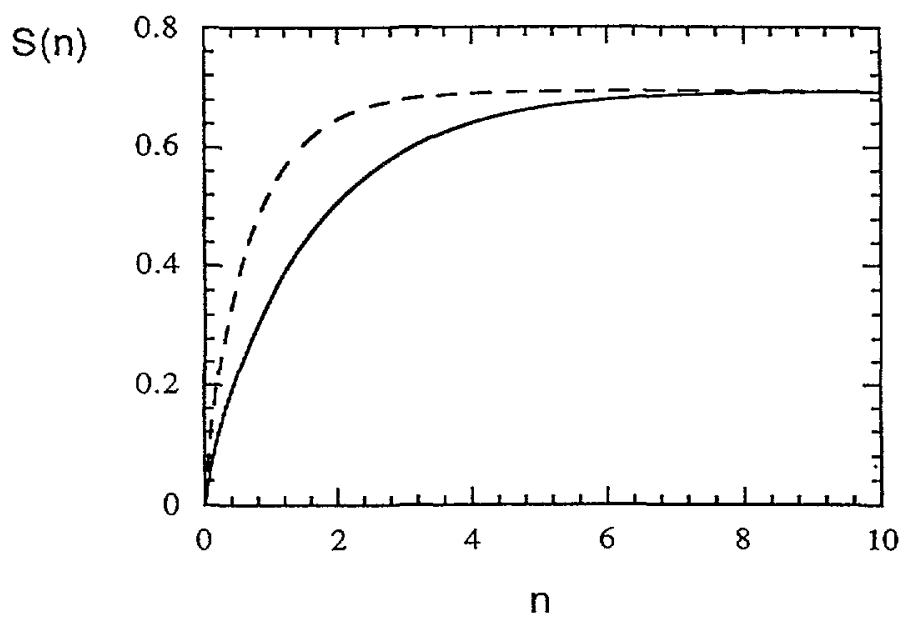

Fig. 2. - Entropy (Eqs. $(4,15)$ ) of radiation plotted as a function of the number of scattering events. Same symbols as in figure 1.

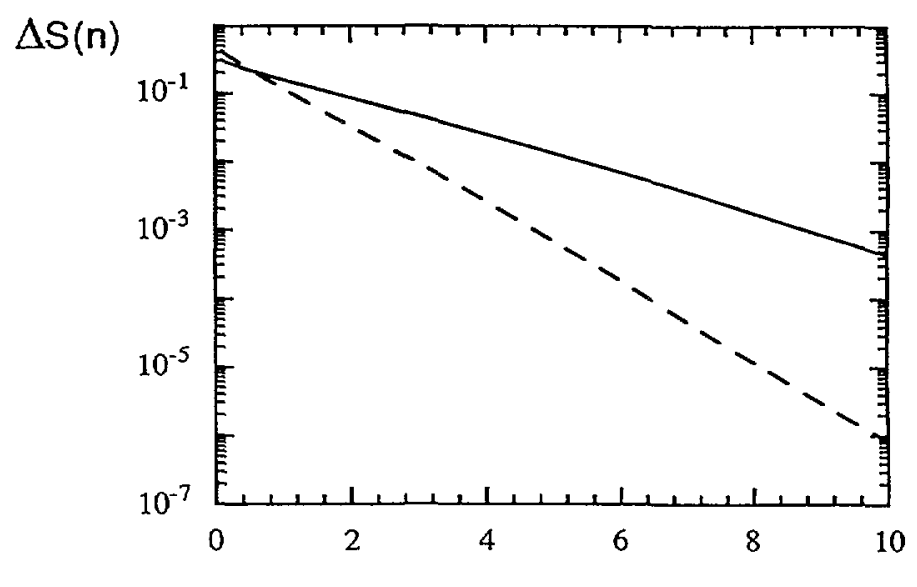

$\mathrm{n}$

Fig. 3. - Entropy production $\Delta S(n)=S(P(n+1))-S(P(n))$ as a function of the number of scattering events. Same symbols as in figure 1 . 
$\Delta S>0$ and $\Delta S=0$, but some problems of this type have been briefly considered by several authors [26-27] (e.g. loading of a spring under the action of a gravitational force, charging of a capacitor, compression of a perfect gas). They found that the irreversibility involved in such physical processes in transforming the system from an initial state to a terminal state bears an inverse relationship to the number of discrete steps in which it is carried out $[26,27]$.

4.2 POLARIZATION DEPENDENCE OF THE TIME AUTOCORRELATION FUnCTION. - Measurement of the autocorrelation of the temporal fluctuations in the intensity of multiply-scattered light by the motion of the scattering centres is termed Diffusing Wave Spectroscopy (DWS). This technique is described in details in $[10,29]$ and was devised to extend the usual quasielastic light scattering to the multiple scattering regime for probing the dynamic structural properties of dense scattering media. Light scattering experiments measure the scattered intensity autocorrelation function defined as $g^{(2)}(t)=\frac{\langle I(t) I(0)\rangle}{\langle I(0)\rangle^{2}}$, where $t$ is a delay time. Treating the field as a zero-mean complex Gaussian process, the auto-correlation functions of the field $g^{(1)}(t)=\frac{\left\langle E(t) E^{*}(0)\right\rangle}{\langle I(0)\rangle}$ and intensity are related through the Siegert factorization theorem : $g^{(2)}(t) \approx 1+\left|g^{(1)}(t)\right|^{2}$. Maret and Wolf [10] related the measurement of the field autocorrelation function $g^{(1)}(t)$ to the Green's function describing the diffusive transport of the intensity. The form of these auto-correlation functions depends on the state of polarization [30]. As was evidenced by MacKintosh et al. [30], each diffusion path leads to a decay of $g^{(1)}(t)$ which depends on the scattering events. For very short times, the correlation function is dominated by long paths for which the waves have lost memory of their incident state of polarization.

In the following, we derive an expression of the degree of polarization for multiply scattered light from a half-space. For the incident pure state of polarization defined by equation (6), the output degree of polarization writes (for $t^{*}<1$ ):

$$
P_{\mathrm{o}}\left(t^{*}\right)=\left\{\left\langle S_{1}\right\rangle^{2} P_{1}^{2}\left(t^{*}\right)+\left\langle S_{2}\right\rangle^{2} P_{2}^{2}\left(t^{*}\right)+\left\langle S_{3}\right\rangle^{2} P_{3}^{2}\left(t^{*}\right)\right\}^{1 / 2},
$$

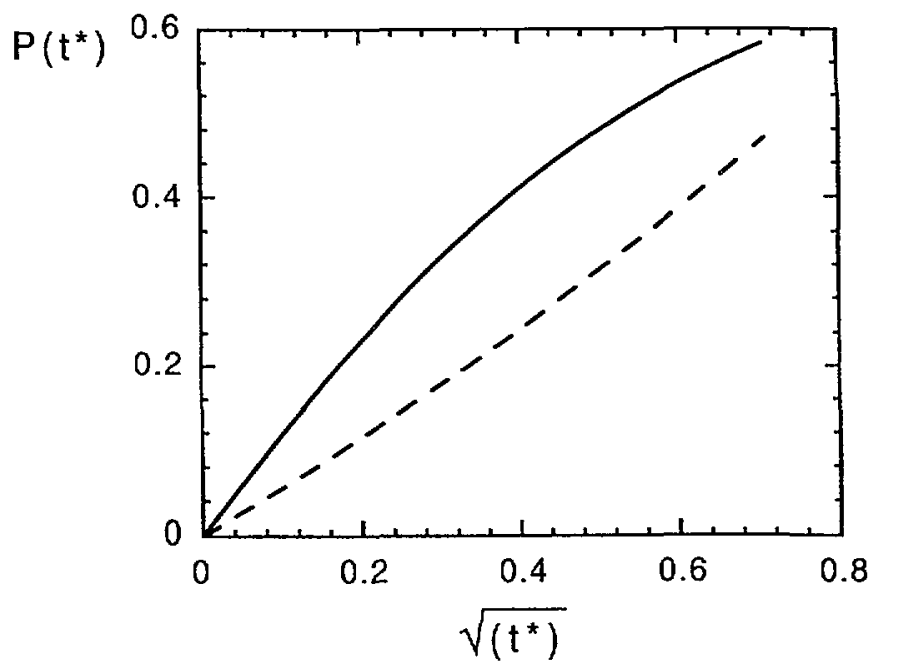

Fig. 4. - Polarization dependence of the autocorrelation functions equation (17) as a function of the dimensionless time $t^{*}$. The curves are linear parallel polarization (solid line), right circular polarization (dashed line). 
where the $P_{j}(t)$ 's $(j=1,2,3)$ are defined in the Appendix B and denote respectively the degree of polarization for linear polarization, linear polarization at $45^{\circ}$ and circular polarization, while $t^{*}$ is the dimensionless time $\left(t / \tau_{0}\right.$ with $\tau_{0}$ being the time required by a scatterer to move one optical wavelength).

As displayed in figure 4, the light is completely depolarized at very short times ( $t^{*} \ll 1$ corresponds to long diffusion paths). Because of the different contributions due to long paths, the $P_{\mathrm{o}}\left(t^{*}\right)$ versus $\left(t^{*}\right)^{1 / 2}$ differ in slope according to the input state of polarization. As before, we find that complete depolarization of circularly polarized incident light requires less scattering events than for the linearly polarized case. This contrasts with longer times (short diffusion paths).

\section{Comment on Freund's reconstruction method.}

To close the paper, we now comment some implications of the recently proposed method [5] of Stokes vector reconstruction after a complete depolarization, to the question of irreversibility. Some representative references about irreversibility in Polarization Optics which we found useful are indicated in [14]. As an historical note, it should be mentioned that Jones [14] was the first to investigate the trade off between entropy, light polarization and irreversibility. Recently Gudkov [14] has been able using Planck's spectral law to calculate the total flux of the spectral entropy of an unpolarized light across the surface of a scattering object. A similar treatment, but generalized to partially polarized light, was put forward by Callies [14]. This author derived a formula for the local entropy production rate by a single scattering using the radiative transfer theory. However these approaches are not easily connected to the multiple scattering situation which is considered here. Given these observations, it is useful to indicate the sense in which irreversibility is featured. Consider a linear interaction between a scattering medium and a plane-wave field. If and only if the entropy remains invariant under the interaction and if no absorption occurs, we shall say the evolution is reversible, otherwise it is irreversible. As a direct consequence, it is easy to prove that any optical transformation characterized by a unitary Mueller matrix (e.g. compensator, rotator) constitutes a reversible operation $[9,14]$. Typical of evolutions that increase the entropy are depolarizing transformations which are irreversible (e.g. multiple scattering). Measurements by selective absorption of states (i.e. filtering) are also irreversible.

Freund [5] considers a diffusively reflected light from a $\mathrm{BaSO}_{4}$ coating (assumed as a random array of point scatterers) which produces complete depolarization of any input totally polarized light (residual degree of polarization $\leqslant 0.03$ ). Reference [5] claims to show that diffusive transport does not lead to any loss of information about the incident polarization state and even after complete depolarization by multiple scattering, the incident state can be reconstructed by a process termed speckle-pattern filtering which he says is very similar to the usual process of determination of the Stokes parameters of a beam of light by filtering [15]. The prediction of Freund would imply that the process is reversible in contradiction with the entropic approach developed here.

We show that the argument is not tenable. The key point here is that the entropy compensation by speckle-pattern filtering suggested by Freund induces a loss of intensity and is therefore irreversible. It is instructive and simple to analyze this by expanding the Stokes vector corresponding to unpolarized light (of intensity $\left\langle S_{0}\right\rangle$ ) in terms of orthogonal polarization states (e.g. right and left circular states each of intensity $\left\langle S_{0}\right\rangle / 2$ ). Now a righthanded circular filter produces both entropy $(-\ln (2))$ and intensity (50\%) losses [9]. Finally we note that a comprehensive discussion of the data produced by Freund [5] could take place only with precise knowledge of both the experimentally determined scattering characteristics 
of the $\mathrm{BaSo}_{4}$ coating and the normalization procedure in the measurement of the Stokes parameters.

\section{Concluding remarks.}

We have considered in some details depolarization and decoherence of a plane-wave field by a weakly disordered and non-absorbing scattering medium. Three main assumptions were introduced : pointlike scattering centers, uncorrelated disorder and diffusion approximation. A typical realization of such medium would be a dense collection of non-interacting spherical scattering particles of size much smaller than the wavelength. On the basis of entropic considerations two main points can be drawn from this paper :

(a) Maximum entropy yields an output totally depolarized light beam vector (large $n$ ) which is similar to that of natural light whose Stokes vector is of the form $\left[\begin{array}{l}1 \\ 0 \\ 0 \\ 0\end{array}\right]$. The results derived in the preceding section do not depend on the specific nature of the scatterers, but rather on the symmetry properties of the scattering medium. Now returning to the question raised in the Introduction, we found that the length scale over which the depolarization and loss of coherence take place is $\zeta=n \ell$, with typical number of scattering events $n$ being about ten. Then for distances larger than $\zeta$, one is justified in describing the transport properties of multiply scattered light by a radiative transfer theory which treat the radiation field as a scalar rather than a four component Stokes vector. In addition, we found that the entropy production falls off exponentially with $\zeta$.

(b) The principle of maximum entropy allows us also to deduce the form of the Mueller matrix elements describing the scattering medium. The explicit calculation of these elements derived from the Bethe-Salpeter equation permitted to test the validity of this approach. In that case a knowledge of the degree of polarization after a double scattering suffices to enable one to construct the Mueller matrix. Specifically, it was seen that full depolarization of linearly polarized light requires more scattering events (typically a factor-of-2) than are required for a circularly polarized light. It is believed that this behavior reflects the specific symmetry properties of $\left\langle S_{3}\right\rangle$; however the details thereof have not been completely disentangled. This can be put in parallel with the results of MacKintosh et al. [31] who investigated the effects of polarization on backscattering of a laser radiation from monodisperse polystyrene spheres in water. These authors have also pointed out that due to the large number of statistically independent contributions, the scattered field is Gaussian distributed by virtue of the central limit theorem. The distribution of Stokes parameters for a Gaussian distributed field has been characterized for both temporal [16, 34, 35] and spatial [36] fluctuations. Reference is also made to a recent paper of Cohen et al. [32] who have recently examined in some details the statistical distribution of the polarization state of multiply scattered optical waves. The ratio of the semi-minor axis to the semi-major axis of the polarization ellipse is denoted by $\varepsilon$ [32]. They found that the distribution function $p(\varepsilon)$ of the ellipticity parameter $\varepsilon$ defining the ellipse of polarization is given by $: p(\varepsilon)=\frac{2\left(1-\varepsilon^{2}\right)}{\left(1+\varepsilon^{2}\right)^{2}}$ indicating that the least probable state is circular polarization (i.e. $\langle\varepsilon\rangle=0.307$ ). A satisfactory agreement was obtained with experimental data [32].

There do of course remain a number of points to be investigated, regarding the fundamental issue of how these results are changed when the dielectric susceptibility of the medium is not completely uncorrelated : a more realistic assumption would be to allow for a finite correlation 
length i.e. Gaussian distribution $\left\langle\mu\left(\mathbf{r}_{1}\right) \mu\left(\mathbf{r}_{2}\right)\right\rangle=\frac{C}{\left(2 \pi \sigma^{2}\right)^{3 / 2}} \exp \left(-\frac{\left|\mathbf{r}_{1}-\mathbf{r}_{2}\right|^{2}}{2 \sigma^{2}}\right)$, where $C$ and $\sigma$ are positive constants; or when the analysis is extended to radiations with a broad bandwidth: i.e. possibility of generating frequency shifts by scattering. Our method of calculating the dependence of the Mueller matrix elements on the number of scattering events gives also rise to interesting possibilities for exploring the behavior of the above in more complicated systems (e.g. scattering from large particles for which the transport of light becomes ballistic [30], extension to less ideal particles shapes which do not scatter light isotropically and involving non-diagonal Mueller matrices).

\section{Acknowledgments.}

One of us (C.B.) is indebted to Prof. Craig. F. Bohren of the Pennsylvania State University for useful correspondence and for bringing some earlier related research to his attention. We are pleased to acknowledge Prof. R. Maynard for critical reading of the manuscript and G. Maret for useful discussions.

\section{Appendix A}

The purpose of this Appendix is to briefly outline the derivation of the correlation function of the field that is needed in section 4 .

The following calculations are more conveniently done in momentum space. Our starting point is the Bethe-Salpeter equation for the field-field correlation function derived from usual diagrammatic expansion $[23,30,37-42]$.

$$
\left\langle G_{l m} G_{j n}^{*}\right\rangle=G_{l m} G_{j n}^{*}+G_{l m^{\prime}} G_{j n^{\prime}}^{*} W_{l j m^{\prime} n^{\prime}} G_{m^{\prime} m} G_{n^{\prime} n}^{*},
$$

where summation over repeated indices is presumed, $G^{*}$ being the complex conjugate of $G$. The first term in the right side of (A1) represents the coherent contribution which goes to zero very rapidly and is neglected while the other term is the incoherent contribution. In the weak scattering situation, the observed intensity is an incoherent sum of contributions of light scattered through all possible paths. For scalar waves, one shows that within the diffusion approximation (i.e. incoherent and diffusive transport of light), the incoherent intensity can be written as $[39,43]$ :

$$
I_{\mathrm{inc}}(\mathbf{r})=\frac{\mathfrak{l}^{2}}{36 \pi^{2}} \sum_{\mathbf{q}} W(\mathbf{q}) \exp (i \mathbf{q} \cdot \mathbf{r})=\frac{6 \pi}{\mathfrak{l}} \sum_{n=1}^{\infty} F(\mathbf{r}, n),
$$

where the Green's function $F(\mathbf{r}, n)$ of the diffusion equation is the weight of diffusion paths and may be obtained by summing successive ladder diagrams.

For a polarized field, the intensity operator $W_{i j m n}$ can be written as :

$$
W_{i j m n}(\mathbf{q})=\frac{6 \pi}{\ell} \Omega \sum_{n=0}^{\infty} \sum_{\alpha}\langle i j \mid \alpha\rangle\left(\lambda_{\alpha}(\mathbf{q})\right)^{n}\langle\alpha \mid m n\rangle,
$$

the first term $n=0$ corresponding to the single scattering situation. The nine ortho-normal eigenvectors $|\alpha\rangle$ and associated eigenvalues $\lambda_{\alpha}(\mathbf{q})$ (to the degree of approximation $O\left(q^{2}\right)$ ) have been derived by a number of authors $[23,30]$ and are not reproduced here. From (A2) and (A3), one finds :

$$
\left\langle G_{l m} G_{j n}^{*}\right\rangle=\frac{3}{2} \sum_{\alpha=1}^{9}\langle i j \mid \alpha\rangle \lambda_{\alpha}(\mathbf{r}, n)\langle\alpha \mid m n\rangle
$$

with

$$
\lambda_{\alpha}(\mathbf{r}, n)=\frac{1}{\Omega} \sum_{\mathbf{q}}\left(\lambda_{\alpha}(\mathbf{q})\right)^{n} \exp (i \mathbf{q} \cdot \mathbf{r}),
$$


and $|i j\rangle,|m n\rangle$ denoting respectively the initial and final states of polarization. Since we are interested to evaluate the dependence of the mean intensity as a function of the scattering number, we write :

$$
\left\langle G_{i m} G_{J n}^{*}\right\rangle=F_{1 j m n}(\mathbf{r}, n) .
$$

As the direction of propagation is along $\mathbf{e}_{z}$, we have :

$$
\begin{gathered}
\left\langle G_{x x} G_{x x}^{*}\right\rangle=\left\langle G_{y y} G_{y y}^{*}\right\rangle=F_{y y y y}=\frac{1}{2}\left[1+2\left(\frac{7}{10}\right)^{n}\right] F\left(\mathbf{r}, n, L_{1}\right), \\
\left\langle G_{x y} G_{x y}^{*}\right\rangle=F_{x x y y}=\frac{1}{2}\left[1-\left(\frac{7}{10}\right)^{n}\right] F\left(\mathbf{r}, n, L_{1}\right), \\
\left\langle G_{x x} G_{y y}^{*}\right\rangle=F_{x y x y}=\frac{3}{4}\left[\left(\frac{7}{10}\right)^{n} F\left(\mathbf{r}, n, L_{2}\right)+\left(\frac{1}{2}\right)^{n} F\left(\mathbf{r}, n, L_{3}\right)\right], \\
\left\langle G_{x y} G_{y x}^{*}\right\rangle=F_{x y y x}=\frac{3}{4}\left[\left(\frac{7}{10}\right)^{n} F\left(\mathbf{r}, n, L_{2}\right)-\left(\frac{1}{2}\right)^{n} F\left(\mathbf{r}, n, L_{3}\right)\right],
\end{gathered}
$$

with the Green's function $F\left(\mathbf{r}, n, L_{l}\right)$ for an infinite medium given by :

$$
F\left(\mathbf{r}, n, L_{l}\right)=\left(\frac{1}{4 \pi L_{i}^{2} n}\right)^{3 / 2} \exp \left\{-\frac{r^{2}}{4 L_{\imath}^{2} n}\right\},
$$

and where we have set $L_{1}^{2}=\frac{\ell^{2}}{3}, L_{2}^{2}=\frac{23 \ell^{2}}{49}$ and $L_{3}^{2}=\frac{3 \ell^{2}}{5}$

It follows at once that carrying out the integration of equations (A7) over the entire space, the $\left\langle G_{l m} G_{j n}^{*}\right\rangle$ are given by the formulae :

$$
\begin{aligned}
\left\langle G_{x x} G_{x x}^{*}\right\rangle=\left\langle G_{y y} G_{y y}^{*}\right\rangle=\frac{1}{2}\left[1+2\left(\frac{7}{10}\right)^{n}\right], \\
\left\langle G_{x y} G_{x y}^{*}\right\rangle=\frac{1}{2}\left[1-\left(\frac{7}{10}\right)^{n}\right], \\
\left\langle G_{x x} G_{y y}^{*}\right\rangle=\frac{3}{4}\left[\left(\frac{7}{10}\right)^{n}+\left(\frac{1}{2}\right)^{n}\right], \\
\left\langle G_{x y} G_{y x}^{*}\right\rangle=\frac{3}{4}\left[\left(\frac{7}{10}\right)^{n}-\left(\frac{1}{2}\right)^{n}\right] .
\end{aligned}
$$

\section{Appendix B}

The purpose of this Appendix is to consider the calculation of the degree of polarization for multiply scattered light within the framework of the DWS. For Brownian scatterers characterized by a diffusion coefficient $D_{\mathrm{B}}$, one shows, in the weak scattering limit and within the diffusion approximation, that the scattered electric field correlation function is given by $[10,30,43]$ :

$$
g^{(1)}(\mathbf{r}, t)=\frac{\left\langle E(0) E^{*}(t)\right\rangle}{\left\langle|E(0)|^{2}\right\rangle}=\frac{\int F(\mathbf{r}, n) \exp \left(-2 n t / \tau_{0}\right) \mathrm{d} n}{\int F(\mathbf{r}, n) \mathrm{d} n}
$$

where $F(\mathbf{r}, n)$ denoting the Green's function of the diffusion equation is the number of scattering paths of length $n \ell$, and $\tau_{0}=k_{0}^{-2} D_{\mathrm{B}}^{-1}$ is the time required by a scatterer to move one 
optical wavelength. From inspection of equation (B1), we note that $g^{(1)}(\mathbf{r}, t)$ is the normalized Laplace transform of $F(\mathbf{r}, n)$, giving then a one to one correspondence between the number of scattering events $n$ and the dimensionless variable $t^{*}=t / \tau_{0}$.

We consider now the special situation of reflected light from a half-space. In this geometry, we assume that the scattering medium occupies the region of space $z>0$ and that the light is incident normally on the semi-infinite medium from the vacuum $z=-\infty$. For a semi-infinite medium, the weight of diffusion paths for scalar waves may be obtained from the above infinite-space case by the method of images [37].

a) LiNEAR POLARIZATION.

Considering an incident field polarized along $\mathbf{e}_{x}$ (labeled $\|$ ), we get for respectively parallel and perpendicularly (labeled $\perp$ ) polarized output light [30] :

$$
\begin{aligned}
& F_{\mathbb{I}}(n)=\langle x x|F| x x\rangle=\left\langle G_{x x} G_{x x}^{*}\right\rangle=\frac{1}{2}\left[1+2\left(\frac{7}{10}\right)^{n}\right] F\left(n, L_{1}\right), \\
& F_{\perp}(n)=\langle x x|F| y y\rangle=\left\langle G_{x y} G_{x y}^{*}\right\rangle=\frac{1}{2}\left[1-\left(\frac{7}{10}\right)^{n}\right] F\left(n, L_{1}\right) .
\end{aligned}
$$

Then, we obtain :

$$
\begin{aligned}
g^{(1)}(t) & =\frac{\exp (-a)+2 \exp (-b)}{1+2 \exp \left(-b^{\prime}\right)} \\
g_{\perp}^{(1)}(t) & =\frac{\exp (-a)-\exp (-b)}{1-\exp \left(-b^{\prime}\right)}
\end{aligned}
$$

where $a=\sqrt{6 t^{*}} ; b=\sqrt{6 t^{*}+3 \ln (10 / 7)}$ and $b^{\prime}=\sqrt{3 \ln (10 / 7)}$.

The degree of polarization is then given by a simple relation :

$$
P_{1}(t)=\frac{\left|g^{(1)}(t)-g_{\perp}^{(1)}(t)\right|}{g \|^{(1)}(t)+g_{\perp}^{(1)}(t)}
$$

\section{b) Circular polarization.}

In a similar manner, the case of circular polarization is developed [30]. The incident unit vector is $\mathbf{E}_{\iota}=\frac{1}{\sqrt{2}}\left(\mathbf{e}_{x}-i \mathbf{e}_{y}\right)$ and the polarization states are :

$$
\begin{aligned}
& |++\rangle=\frac{1}{2}\{|x x\rangle-i|y x\rangle+i|x y\rangle+|y y\rangle\}, \\
& |+-\rangle=\frac{1}{2}\{|x x\rangle+i|y x\rangle-i|x y\rangle+|y y\rangle\} .
\end{aligned}
$$

In passing, we would like to remark the permutation symmetry of these vectors under the conjugation operation.

We call $F_{+}$(resp. $F_{-}$) the Green's function corresponding to the positive (resp. negative) helicity. In like fashion as above we have :

$$
\begin{aligned}
& F_{+}=\langle++|F|++\rangle, \\
& F_{-}=\langle+-|F|++\rangle \text {, } \\
& \left.\begin{array}{l}
F_{+} \\
F_{-}
\end{array}\right\}=\frac{1}{2}\left\{\left\langle G_{x x} G_{x x}^{*}\right\rangle+\left\langle G_{x y} G_{x y}^{*}\right\rangle \pm\left\langle G_{x x} G_{y y}^{*}\right\rangle-( \pm)\left\langle G_{x y} G_{y x}^{*}\right\rangle\right\}
\end{aligned}
$$


with

$$
\left.\begin{array}{l}
F_{-}(n) \\
F_{+}(n)
\end{array}\right\}=\frac{1}{4}\left[2+\left(\frac{7}{10}\right)^{n} \pm \frac{5}{3}\left(\frac{1}{2}\right)^{n}\right] F\left(n, L_{1}\right)
$$

The corresponding temporal correlation functions are :

$$
\left.\begin{array}{l}
g_{-}^{(1)}(t) \\
g_{+}^{(1)}(t)
\end{array}\right\}=\frac{2 \exp (-a)+\exp (-b) \pm \frac{5}{3} \exp (-d)}{2+\exp \left(-b^{\prime}\right) \pm \frac{5}{3} \exp \left(-d^{\prime}\right)},
$$

with $a, b, b^{\prime}$ given by the aforementioned expressions and $d=\sqrt{(5 / 3)\left(2 t^{*}+\ln (2)\right)}$; $d^{\prime}=\sqrt{(5 / 3) \ln (2)}$. The corresponding degree of polarization writes :

$$
P_{3}(t)=\frac{\left|g_{+}^{(1)}(t)-g_{-}^{(1)}(t)\right|}{g_{+}^{(1)}(t)+g_{-}^{(1)}(t)}
$$

Similarly, one gets for a polarization state at $45^{\circ}$

$$
P_{2}(t)=\frac{\left|g_{\| 1}^{(1)}(t)-g_{\perp 1}^{(1)}(t)\right|}{g_{\| 1}^{(1)}(t)+g_{\perp 1}^{(1)}(t)},
$$

with

$$
\left.\begin{array}{l}
g_{1}^{(1)}(t) \\
g_{\perp 1}^{(1)}(t)
\end{array}\right\}=\frac{2 \exp (-a)+\exp (-b) \pm \frac{49}{23} \exp (-c)}{2+\exp \left(-b^{\prime}\right) \pm \frac{49}{23} \exp \left(-c^{\prime}\right)},
$$

and $c=\sqrt{(49 / 23)\left(2 t^{*}+\ln (10 / 7)\right)} ; c^{\prime}=\sqrt{(49 / 23) \ln (10 / 7)}$.

These results may be compared with those of reference $[30]$ : for this purpose we indicate that the slopes of $P_{1}(t)$ and $P_{3}(t) v s$. $\sqrt{6 t^{*}}$ taken at the origin are respectively equal to $\left(\gamma_{\perp}-\gamma_{\|}\right) / 2$ and $\left(\gamma_{+}-\gamma_{-}\right) / 2$, in the notations of reference [30]. From figure 4 we note also that the ratio of these slopes is equal to 2.2 , i.e. close to the value discussed in section 4.1 .

\section{References}

[1] SHENG P., Scattering and Localization of Classical Waves in Random Media (World Scientific, Singapore, 1990). For a presentation of some analogies between electron and optical wave phenomena, reference is also made to : KaveH M., Physica B 175 (1991) 1. The connection between these two sets of problems has been also developed in a lucid presentation by FENG $S$., LEE P. A., Science 251 (1991) 633.

[2] Ishimaru A., Wave Propagation and Scattering in Random Media (Academic Press, New York, 1978).

[3] See the feature issue on wave propagation and scattering in random media, J. Opt. Soc. Am. A 2 (1985) 206 and references therein.

[4] FREUnd I., Opt. Commun. 86 (1991) 216.

[5] FREUND I., Opt. Lett. 15 (1990) 1425.

[6] Freund I., Opt. Commun. 81 (1991) 251. This treatment follows Akkermans E., Ph. D. Thesis, Fourier University, Grenoble, France (1986).

[7] KAVEH M., Waves in Random Media 3 (1991) S121. 
[8] Freund I., Kaveh M., Berkovits R., Rosenbluh M., Phys. Rev. B 42 (1990) 2613.

See also: Freund I., Rosenbluh M., Berkovits R., Phys. Rev. B 39 (1989) 12403.

[9] Brosseau C., Optik 88 (1991) 109.

[10] Maret G., Wolf P. E., Z. Phys. B 65 (1987) 409.

See also Rosenbluh M., Hoshen M., Freund I., Kaveh M., Phys. Rev. Lett. 58 (1987) 2754.

[11] Wolf E., Foley J. T., GoRi F., J. Opt. Soc. Am. 6 (1989) 1142.

[12] Born M., Wolf E., Principles of Optics (Pergamon Press, Oxford, 1980).

[13] For an account of the theory of partial coherence formulated in terms of spatial-time correlation functions, see, for example, Mandel L., Wolf E., Rev. Mod. Phys. 37 (1965) 231 ;

See also, Wolf E., J. Opt. Soc. Am. 72 (1982) 343 for presentation of a theory of partial coherence in the space-frequency domain.

[14] Jones R. C., J. Opt. Soc. Am. 43 (1953) 138 ;

See also GudKov N. D., Opt. Spektrosk. 68 (1990) 224 (Opt. Spectrosc. USSR 68 (1990) 130) and Callies U., Beitr. Phys. Atmosph. 62 (1989) 212.

[15] Azzam R. M. A., Bashara N. B., Ellipsometry and Polarized Light (North-Holland, Amsterdam, 1977).

[16] Barakat R., J. Opt. Soc. Am. 4 (1987) 1256.

[17] BLuM K., Density Matrix Theory and Applications (Plenum Press, New York, 1981).

[18] Prigogine I., Thermodynamic of Irreversible Processes (Wiley, New York, 1951).

[19] Perrin F., J. Chem. Phys. 10 (1942) 415.

[20] VAN DE Hulst H. C., Light Scattering by Small Particles (Wiley, New York, 1957) p. 40.

[21] Hovenier J. W., J. Atm. Sci. 26 (1969) 488.

[22] Freund I., Waves in Random Media 1 (1991) 245.

[23] Stephen M. J., Cwilich G., Phys. Rev. B 34 (1986) 7564 ;

See also Stephen M. J., Phys. Rev. B 37 (1988) 1.

[24] Bourret R., Opt. Acta 21 (1974) 721.

[25] LyOYd S., Phys. Rev. A 39 (1989) 5378.

[26] Calkin M. G., Krang D., Am. J. Phys. 51 (1983) 78.

[27] See also Gupta V. K., Shanker G., Sharma N. K., Am. J. Phys. 52 (1984) 945 ;

HEINRICH F., Am. J. Phys. 54 (1986) 742.

[28] Berne B. J., Pecora R., Dynamic Light Scattering (Wiley, New York, 1976).

[29] Pine D. J., Weitz D. A., Chaikin P. M., Herbolzheimer E., Phys. Rev. Lett. 60 (1988) 1134.

[30] Mackintosh F. C., John S., Phys. Rev. B 40 (1989) 2383.

[31] Mackintosh F. C., Zhu J. X., Pine D. J., Weitz D. A., Phys. Rev. B 40 (1989) 9342.

[32] Cohen S. M., Elryahu D., Freund I., Kaveh M., Phys. Rev. A 43 (1991) 5748.

[33] Brosseau C., Barakat R., Rockower E. B., Opt. Commun. 82 (1991) 204.

[34] Saleh B. E. A., Photoelectron Statistics (Springer, Berlin, 1978).

[35] Brosseau C., Barakat R., Opt. Commun. 84 (1991) 127.

[36] Steeger P. F., Fercher A. F., Optica Acta 29 (1982) 1395.

[37] Morse P. M., Feshbach H., Methods in Theoretical Physics (McGraw-Hill, New York, 1953) vol. 1.

[38] Abrikosov A. A., Gor'kov L. P., DzIaloshinskir I. E., Methods of Quantum Field Theory in Statistical Physics (Prentice-Hall, New York, 1963).

[39] Akkermans E., Wolf P. E., Maynard R., Maret G., J. Phys. France 49 (1988) 77.

[40] Golubentzev A. A., Zh. Eksp. Teor. Fiz. 86 (1984) 47 (Sov. Phys. JETP 59 (1984) 26).

See also Golubentzev A. A., Zh. Eksp. Teor. Fiz. 96 (1989) 1933 (Sov. Phys. JETP 69 (1989) 1090).

[41] Barabanenkov Y. N., Sov. Phys. Usp. 18 (1975) 673.

[42] LeE J. K., Kong J. A., J. Opt. Soc. Am. A 2 (1985) 2171.

[43] Bicout D., Akkermans E., Maynard R., J. Phys. I France 1 (1991) 471. 